Erratum

\title{
All the physical and electrical parameters of the MOS transistor on a single graph $(\mathrm{Q} \Psi)$
}

G. Vincent ${ }^{\mathrm{a}}$

Département de Physique, Université Joseph Fourier, Grenoble, France

Laboratoire de Technologie de la Microélectronique, 17 avenue des Martyrs, 38054 Grenoble 9, France

Eur. Phys. J. Appl. Phys. 32, 155 (2005)

Received 11 March 2006

Published online 2006 - (c) EDP Sciences

In Section Conclusion (p. 158), the first equation should read:

$$
I=\mu_{n} \frac{W}{L} \frac{\varepsilon_{o x}}{e_{o x}}\left[\left(V_{G}-2 \psi_{b}-\frac{V_{D}}{2}\right) V_{D}-\frac{2}{3} \frac{\sqrt{2 \varepsilon_{S C} q N_{a}}}{\frac{\varepsilon_{o x}}{e_{o x}}}\left\{\left(V_{D}+2 \psi_{b}+\left|V_{s u b}\right|\right)^{\frac{3}{2}}-\left(2 \psi_{b}+\left|V_{s u b}\right|\right)^{\frac{3}{2}}\right\}\right]
$$

a e-mail: gilbert.belledonne@wanadoo.fr 